\title{
The Roman Domination Problem in Unit Disk Graphs ${ }^{\star}$
}

\author{
Weiping Shang and Xiaodong Hu \\ Institute of Applied Mathematics, Chinese Academy of Sciences \\ P. O. Box 2734, Beijing 100080, China \\ \{shangwp, xdhu\}@amss.ac.cn
}

\begin{abstract}
Unit disk graphs are the intersection graphs of equal sized disks in the plane, they are widely used as a mathematical model for wireless ad-hoc networks and some problems in computational geometry. In this paper we first show that the Roman domination problem in unit disk graphs is NP-hard, and then present a simple linear time approximation algorithm and a polynomial-time approximation scheme for this problem, respectively.
\end{abstract}

Keywords: Domination, approximation algorithm, unit disk graph.

\section{Introduction}

Let $G=(V, E)$ be a simple and undirected graph. A set $D \subseteq V$ is a dominating set if every vertex in $V \backslash D$ is adjacent to at least one vertex in $D$. The domination number, denoted $\gamma(G)$, is the minimal cardinality of a dominating set in $G$. The minimum dominating set problem is to compute a dominating set of minimal cardinality for any given graph $G$. A Roman dominating function 1 of a graph $G$ is defined as a function $f: V(G) \rightarrow\{0,1,2\}$ satisfying the condition that every vertex $u$ with $f(u)=0$ is adjacent to at least one vertex $v$ with $f(v)=$ 2. The weight of a Roman dominating function is $f(V)=\sum_{v \in V} f(v)$. The Roman domination number, denoted $\gamma_{R}(G)$, is the minimum weight of Roman dominating functions in $G$. The minimum Roman domination problem is to compute a minimum weight dominating function for any given graph $G$.

Minimum Roman domination problem is introduced in [1] as one of variants of classical dominating set problem. It comes with a nice story: The Roman Empire needs to defence itself by positioning some legions on the various parts of the Empire in such a way that either (1) a specific region $v$ is also the location of at least one legion or (2) one region $u$ neighboring $v$ has two legions, so that $u$ can afford sending off one army to the region $v$ (in case of an attack) without loosing self-defence capabilities. Since it is expensive to maintain a legion at a location, the Emperor would like to station as few legions as possible, while still defending

\footnotetext{
* This work was supported in part by the National Natural Science Foundation of China under Grant No. 70221001 and 10531070.
} 
the Roman Empire. Roman domination problem has not only historical but also mathematical interests, particularly in the field of server placements [3].

In reality, it is desired that dispatching a legion from one location to another location should not take too much time; In particular, it is desirable that a legion from one location could be dispatched to another location if the Euclidean distance between the two corresponding points is no more than a specified bound. Unit disk graphs satisfy such a property. A undirected graph $G=(V, E)$ is a unit disk graph (UDG) if its vertices can be put in one-to-one correspondence with disks of equal radius in the plane in such a way that two vertices are joined by an edge if and only if the corresponding disks intersect. In other words, $G$ is a UDG if there exists a map $f: V \rightarrow R^{2}$ such that $(u, v) \in E$ if and only if $\|f(u)-f(v)\| \leq 1$, where $\|\cdot\|$ denotes the Euclidean norm. Grid graphs compose a subclass of unit disk graphs under such an intersection model that all the disks have centers with integer coordinates and radius $1 / 2$.

It has been shown in [7] that many graph related problems in unit disks graphs are NP-hard, including the independent set problem, the dominating set and connected dominating set problems; In fact, the dominating set problem is NP-hard even for grid graphs. Polynomial-Time Approximation Schemes (PTASs) for these problems in unit disk graphs are presented in 10, 11. Roman domination problem in general graphs is proved to be NP-hard in [2], $(2+2 \ln (n))$ approximation algorithms and a PTAS are proposed in 3. for general graphs and for minimum planar Roman domination problem, respectively.

In this paper, we consider the Roman domination problem in unit disk graphs. We will first give some preliminaries about the problem in Section 2, and then we will prove that the Roman domination problem in unit disk graphs is NP-hard in Section 3, after that we will propose a simple linear-time approximation algorithm and a polynomial-time approximation scheme for the problem in Section 4. In Section 5 we conclude the paper.

\section{Preliminaries}

Let $G$ be a graph with vertex-set $V(G)$ and edge-set $E(G)$. For any vertex $v \in V$, the closed neighborhood of $v$ is denoted and defined by $N(v) \equiv\{u \in V(G): u v \in$ $E(G)\} \cup\{v\}$. For a subset $S$ of $V$, let $N(S)=\bigcup_{v \in S} N(v)$, and for a natural number $r$, define the $r$-th neighborhood of $v \in V$ as $N^{r}(v) \equiv N\left(N^{r-1}(v)\right)$, where $N^{1}(v)=N(v)$.

A subset $U \subseteq V$ is an independent set (IS) of $G$ if all vertices in $U$ are pairwise non-adjacent, and a maximal independent set (MIS) $U$ of $G$ is such an IS that each vertex $V \backslash U$ is adjacent to at least one vertex in $U$. Clearly, an MIS $I(G)$ of $G$ is also a dominating set of $G$. Thus any minimum dominating set $D(G)$ of $G$ has size no more than that of an MIS $I(G)$. In addition, a subset $S$ of $V(G)$ is called a 2-packing if $N(u) \cap N(v)=\emptyset$ for every pair of vertices $u, v \in S$.

Some useful facts on Roman dominating functions were proved in [1, which will be used in our following discussions. 
Proposition 1. For any graph $G, \gamma(G) \leq \gamma_{R}(G) \leq 2 \gamma(G)$.

Proposition 2. Let $f$ be a minimum Roman dominating function of a graph $G$ without isolated vertices. Let $V_{i}$ be the sets of vertices $v$ with $f(v)=i$ for $i=0,1,2$. Let $f=\left(V_{0}, V_{1}, V_{2}\right)$ be such a function that $\left|V_{1}\right|$ is minimal. Then $V_{1}$ is a 2-packing and there is no edge between $V_{1}$ and $V_{2}$.

Proposition 3. For any non-trivial connected graph $G, \gamma_{R}(G)=\min \{2 \gamma(G \backslash$ $S)+|S|: S$ is a 2-packing $\}$.

Clearly, a function $f=\left(V_{0}, V_{1}, V_{2}\right)$ is a Roman dominating function if the set $V_{2}$ dominates the set $V_{0}$, and the weight of $f$ is $f(V)=\sum_{v \in V} f(v)=2\left|V_{2}\right|+$ $\left|V_{1}\right|$. Moreover, a Roman dominating function $f=\left(V_{0}, V_{1}, V_{2}\right)$ defines a Roman dominating set $D_{R}=\left(V_{1}, V_{2}\right)$ since, for each vertex $v \in V$, we have either $v \in$ $V_{1} \cup V_{2}$ or $v$ is dominated by some node in $V_{2}$. The weight of a Roman dominating set $D_{R}$ is $W\left(D_{R}\right)=2\left|V_{2}\right|+\left|V_{1}\right|$. If $D_{R}$ is a minimum Roman dominating set (MRDS) of graph $G$, then $W\left(D_{R}\right)=\gamma_{R}(G)$.

\section{Complexity Study of Roman Domination Problem}

Let $V=\left\{v_{1}, v_{2}, \cdots, v_{n}\right\}$ be the set of points located in the Euclidean plane. Denote the coordinates of $v_{i}$ by $\left(x_{i}, y_{i}\right)$ for $i=1,2, \cdots, n$. For each vertex $v_{i} \in V$, there is a vertex $u_{i}$ with coordinates $\left(x_{i}+\frac{1}{10}, y_{i}\right)$, and let $U=\left\{u_{1}, u_{2}, \cdots, u_{n}\right\}$ denote the set associated with $V$. Now construct a graph $G^{\prime}=\left(V^{\prime}, E^{\prime}\right)$ with vertex-set $V^{\prime}=V \cup U$ and edge-set $E^{\prime}=\left\{(u, v): u, v \in V^{\prime}\right.$ and $\left.\|u v\| \leq 1+\frac{1}{10}\right\}$. It is clear that $G^{\prime}$ is a unit disk graph, and $N\left(v_{i}\right)=N\left(u_{i}\right)$ in graph $G^{\prime}$. Moreover, the following lemmas give some other properties of graph $G^{\prime}$.

Lemma 1. Let $f^{\prime}=\left(V_{0}^{\prime}, V_{1}^{\prime}, V_{2}^{\prime}\right)$ be a minimal Roman dominating function of $G^{\prime}=\left(V^{\prime}, E^{\prime}\right)$. Then $\left|\left\{u_{i}, v_{i}\right\} \cap V_{2}^{\prime}\right| \leq 1$, for $i=1,2, \cdots, n$.

Proof. Suppose, by contradiction, that there exists a $j$ such that $\left\{u_{j}, v_{j}\right\} \subseteq V_{2}$. Let $V_{0}^{\prime \prime}=V_{0}^{\prime} \cup\left\{u_{j}\right\}, V_{1}^{\prime \prime}=V_{1}^{\prime}$ and $V_{2}^{\prime \prime}=V_{2}^{\prime} \backslash\left\{u_{j}\right\}$. It is easy to verify that function $f^{\prime \prime}=\left(V_{0}^{\prime \prime}, V_{1}^{\prime \prime}, V_{2}^{\prime \prime}\right)$ is a Roman dominating function satisfying $f^{\prime \prime}\left(V^{\prime}\right)<f^{\prime}\left(V^{\prime}\right)$, a contradicting that $f^{\prime}$ is minimal.

Lemma 2. Let $f^{\prime}=\left(V_{0}^{\prime}, V_{1}^{\prime}, V_{2}^{\prime}\right)$ be a minimal Roman dominating function of $G^{\prime}=\left(V^{\prime}, E^{\prime}\right)$. Then there exists a minimal Roman dominating function $f^{\prime \prime}=\left(V_{0}^{\prime \prime}, V_{1}^{\prime \prime}, V_{2}^{\prime \prime}\right)$ satisfying $f^{\prime \prime}\left(V^{\prime}\right)=f^{\prime}\left(V^{\prime}\right)$ such that $V_{1}^{\prime \prime}=\emptyset$ and $V_{2}^{\prime \prime} \cap$ $\left\{u_{1}, u_{2}, \ldots, u_{n}\right\}=\emptyset$.

Proof. Suppose that $V_{1}^{\prime} \neq \emptyset$. Then let $v_{j} \in V_{1}^{\prime}$ for some $j$. Thus we have $N\left(v_{j}\right) \cap V_{2}^{\prime}=\emptyset$ and $N\left(u_{j}\right) \cap V_{2}^{\prime}=\emptyset$ since $N\left(v_{j}\right)=N\left(u_{j}\right)$, which implies $u_{j} \in V_{1}^{\prime}$. Hence we have either $\left|\left\{u_{i}, v_{i}\right\} \cap V_{1}\right|=0$ or 2 for each $i$. Let $V_{1}^{\prime}=$ $\left\{v_{i 1}, u_{i 1}, v_{i 2}, u_{i 2}, \ldots, v_{i k}, u_{i k}\right\}$ for some $1 \leq k \leq n, V_{0}^{\prime \prime}=V_{0}^{\prime} \cup\left\{u_{i 1}, u_{i 2}, \ldots, u_{i k}\right\}$, $V_{1}^{\prime \prime}=\emptyset$, and $V_{2}^{\prime \prime}=V_{2}^{\prime} \cup\left\{v_{i 1}, v_{i 2}, \ldots, v_{i k}\right\}$. Clearly, $f^{\prime \prime} \equiv\left(V_{0}^{\prime \prime}, V_{1}^{\prime \prime}, V_{2}^{\prime \prime}\right)$ is a Roman dominating function satisfying $f^{\prime \prime}\left(V^{\prime}\right)=f^{\prime}\left(V^{\prime}\right)$. 
Now suppose that $V_{2}^{\prime} \cap\left\{u_{1}, u_{2}, \cdots, u_{n}\right\} \neq \emptyset$. Assume, without loss of generality, that $V_{2}^{\prime} \cap\left\{u_{1}, u_{2}, \cdots, u_{n}\right\}=\left\{u_{1}, u_{2}, \cdots, u_{m}\right\}$ for some $1 \leq m \leq n$. Since $\left|\left\{u_{i}, v_{i}\right\} \cap V_{2}^{\prime}\right| \leq 1$ for each $i$, then $\left\{v_{1}, v_{2}, \cdots, v_{m}\right\} \subseteq V_{0}^{\prime}$. Let $V_{0}^{\prime \prime}=\left(V_{0}^{\prime} \backslash\right.$ $\left.\left\{v_{1}, v_{2}, \cdots, v_{m}\right\}\right) \cup\left\{u_{1}, u_{2}, \cdots, u_{m}\right\}, V_{1}^{\prime \prime}=V_{1}^{\prime}$ and $V_{2}^{\prime \prime}=\left(V_{2}^{\prime} \backslash\left\{u_{1}, u_{2}, \cdots, u_{m}\right\}\right)$ $\cup\left\{v_{1}, v_{2}, \cdots, v_{m}\right\}$. It is easy verify that $f^{\prime \prime} \equiv\left(V_{0}^{\prime \prime}, V_{1}^{\prime \prime}, V_{2}^{\prime \prime}\right)$ is a Roman dominating function satisfying $f^{\prime \prime}\left(V^{\prime}\right)=f^{\prime}\left(V^{\prime}\right)$ and $V_{2}^{\prime \prime} \cap\left\{u_{1}, u_{2}, \ldots, u_{n}\right\}=\emptyset$. The lemma is then proved.

Theorem 1. The minimum Roman dominating set problem in unit disk graphs is NP-hard.

Proof. The reduction is from the minimum dominating set problem in grid graphs, which is known to be NP-hard. Given a grid graph $G=(V, E)$, we will construct a unit disk graph $G^{\prime}=\left(V^{\prime}, E^{\prime}\right)$ such that $G$ has a dominating set $D$ with $|D| \leq k$ if and only if $G^{\prime}$ has a Roman dominating set $D_{R}$ with $W\left(D_{R}\right) \leq 2 k$.

Given any grid graph $G=(V, E)$ with vertex-set $V=\left\{v_{1}, v_{2}, \ldots, v_{n}\right\}$, where each $v_{i}$ has integral coordinates $\left(x_{i}, y_{i}\right)$ and $\left(v_{i}, v_{j}\right) \in E$ if and only if $\left\|v_{i} v_{j}\right\|=1$. For each vertex $v_{i} \in V$, we add a new vertex $u_{i}$ with coordinates $\left(x_{i}+\frac{1}{10}, y_{i}\right)$. Now construct the graph $G^{\prime}=\left(V^{\prime}, E^{\prime}\right)$ with vertex-set $V^{\prime}=\left\{v_{1}, u_{1}, v_{2}, u_{2}, \ldots, v_{n}, u_{n}\right\}$, and for any two vertices $u, v \in V^{\prime},(u, v) \in E^{\prime}$ if and only if $\|u v\| \leq 1+1 / 10$. It is clear that $G^{\prime}$ is a unit disk graph, and for each $1 \leq i \leq n, v_{i}$ and $u_{i}$ have the same neighborhood $N\left(v_{i}\right)=N\left(u_{i}\right)$, and the same degree $d_{G^{\prime}}\left(u_{i}\right)=d_{G^{\prime}}\left(v_{i}\right)=$ $2 d_{G}\left(v_{i}\right)+1$.

Let $D_{R}$ be a minimal Roman dominating set of $G^{\prime}$. Then by Lemma 2, we can assume, without loss of generality, that $D_{R}=\left(\emptyset, V_{2}\right)$ and $V_{2} \cap\left\{u_{1}, u_{2}, \cdots, u_{n}\right\}=$ $\emptyset$. Suppose that $D$ is a dominating set of $G$ with $|D| \leq k$. Let $D_{R}=(\emptyset, D)$, then $D_{R}$ is a Roman dominating set with $W\left(D_{R}\right) \leq 2 k$. Conversely, suppose that $G^{\prime}$ has a Roman dominating set $D_{R}=\left(\emptyset, V_{2}\right), V_{2} \cap\left\{u_{1}, u_{2}, \ldots, u_{n}\right\}=\emptyset$ and $W\left(D_{R}\right) \leq 2 k$. Then $V_{2}$ is also a dominating set of $G,\left|V_{2}\right|=\frac{1}{2} W\left(D_{R}\right) \leq k$. Let $D=V_{2}$, then $G$ has a dominating set $D$ with $|D| \leq k$. Therefore, $G$ has a dominating set $D$ with $|D| \leq k$ if and only if $G^{\prime}$ has a Roman dominating set $D_{R}$ with $W\left(D_{R}\right) \leq 2 k$. The proof is then completed.

\section{Approximation Algorithms}

It is well known that any maximal independent set $D$ is also a dominating set. Thus $D_{R}=(\emptyset, D)$ is a Roman dominating set. One method of finding a Roman dominating set in a graph is to find a maximal independent set. A straightforward method of finding a maximal independent set is as follows: Select an arbitrary vertex $v \in V$, add $v$ to the current independent set $D$, which is initially set to be an empty set, and delete $N(v)$ from the current graph $G$, and then repeat the process until all vertices in $V(G)$ have been deleted. 
Algorithm A. 5-Approximation Algorithm for MRDS

Input: A unit disk graph $G=(V, E)$

Output: A Roman dominating set $D_{R}$

1. $S:=V, D:=\emptyset$

2. while $S \neq \emptyset$ do

3. Choose $v \in S$

4. $\quad D:=D \cup\{v\}, S:=S \backslash N(v)$

5. end-while

6. return $D_{R}=(\emptyset, D)$

Theorem 2. Let $G=(V, E)$ be a unit disk graph, Algorithm A returns a Roman dominating set in time of $O(|E|)$ whose size is at most five times that of the minimum Roman dominating set.

Proof. It is clear that the algorithm returns a Roman dominating set in time of $O(|E|)$. Let $D_{R}^{*}=\left(V_{1}^{*}, V_{2}^{*}\right)$ denote an optimal Roman dominating set of $G$ such that $V_{1}^{*}$ is a minimal. By Proposition 2 we have $V_{1}^{*}$ is a 2-packing and there is no edge between $V_{1}^{*}$ and $V_{2}^{*}$. Note that $G$ is a unit disk graph and the returned set $D$ is an independent set. Moreover, no vertex in $V_{2}^{*}$ can dominate more than 5 vertices in $D \backslash V_{1}^{*}$, and each vertex in $V_{1}^{*}$ only can dominate itself. Hence we have

$$
\left|V_{2}^{*}\right| \geq \frac{|D|-\left|V_{1}^{*} \cap D\right|}{5}
$$

and

$$
\gamma_{R}(G)=f(V)=2\left|V_{2}^{*}\right|+\left|V_{1}^{*}\right| \geq \frac{2|D|}{5}=\frac{W\left(D_{R}\right)}{5},
$$

and then the theorem follows.

In the following we will show that there exists an algorithm that can return a better approximation solution at the expenses of increasing the time complexity. For this purpose, we first show the following lemma.

Lemma 3. If the maximum independent set $I(G)$ of graph $G=(V, E)$ has cardinality $|I(G)|$ no greater than a constant $c$. Then $\gamma_{R}(G)$ can be computed in polynomial time.

Proof. By $|I(G)| \leq c$, we have $\gamma(G) \leq c$ since any maximal independent set is also a dominating set. As $\gamma_{R}(G)=\min \{2 \gamma(G-S)+|S|: S$ is a 2-packing $\}$, $\gamma(G \backslash S)$ and $|S|$ both are at most $c$. Thus to compute $\gamma_{R}(G)$ we can first choose a 2-packing set $S$ in $G$, and then find a minimum dominating set in $G \backslash S$, finally choose the set $S$ such that $|S|+2 \gamma(G-S)$ is minimal. Clearly we can compute $\gamma_{R}(G)$ by a brute-force method in time of $O\left(|V|^{c}\right)$.

The basic idea of our polynomial time approximation scheme is as follows: First, compute a local Roman dominating set for a neighborhood of a vertex, and 
then expand this neighborhood until we have formed some sets that satisfy the desired bound. Secondly, eliminate the current neighborhoods and repeat the same operation for the remaining graph.

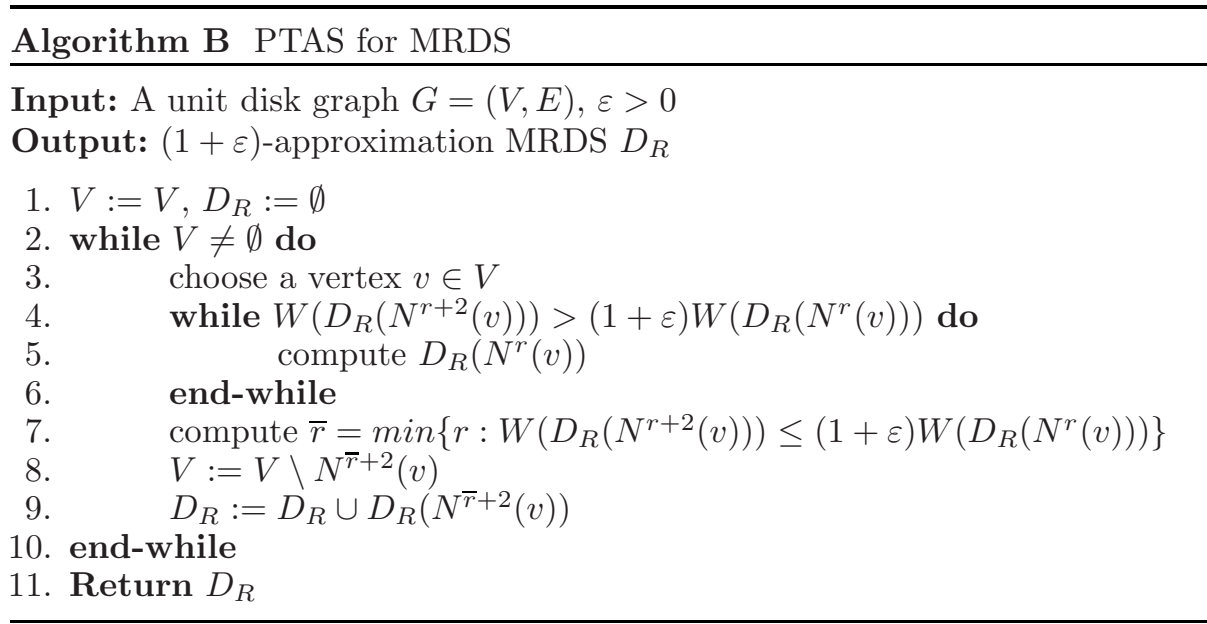

Let $k$ be the total number of executed iterations by Algorithm B. Let $v_{1}, v_{2}$, $\cdots, v_{k}$ be the vertices chosen in step 3 , and $N_{i}, i=1,2, \cdots, k$, denote the corresponding neighborhoods, i.e., $N_{i}=N^{\bar{r}_{i}+2}\left(v_{i}\right)$. By the rules of Algorithm B, it is easy to verify that the returned set $D_{R}=\bigcup_{i=1}^{k} D_{R}\left(N_{i}\right)$ is a Roman dominating set of $G$. Moreover, we have the following lemma.

Lemma 4. Let $G=(V, E)$ be a unit disk graph. Then $W\left(D_{R}\left(N^{r}(v)\right)\right) \leq 8(r+$ $1 / 2)^{2}$ for every $v \in V$ and any positive integer $r$.

Proof. From the definition of a unit disk graph, we know that for any $w \in N^{r}(v)$, the Euclidean distance between $w$ and $v$ is at most $r$. Let $I\left(N^{r}(v)\right)$ denote an independent set in $N^{r}(v)$. Then the sets of unit-diameter disks centered at the nodes in $I\left(N^{r}(v)\right)$ are disjoint and their convex hull is contained in the disk of radius $r+1 / 2$. Then $\left|I\left(N^{r}(v)\right)\right| \leq 4(r+1 / 2)^{2}$, and $W\left(D_{R}\left(N^{r}(v)\right)\right) \leq$ $2\left|D\left(N^{r}(v)\right)\right| \leq 2\left|I\left(N^{r}(v)\right)\right|$. Thus the lemma follows.

Lemma 5. The obtained $\bar{r}$ in Step 7 of Algorithm B is no greater than constant $c=O\left(\frac{1}{\varepsilon^{2}} \ln \frac{1}{\varepsilon}\right)$.

Proof. Note that $W\left(D_{R}\left(N^{0}(v)\right)\right)=1$ and $W\left(D_{R}\left(N^{1}(v)\right)\right)=2$ since vertex $v$ dominates itself and all its neighbors. For an arbitrary value $r<\bar{r}$, consider the following two cases.

Case (1) $r$ is an even number. By the rules of Algorithm B and Lemma 4 we have

$$
8\left(r+\frac{1}{2}\right)^{2} \geq W\left(D_{R}\left(N^{r}(v)\right)\right)
$$




$$
\begin{aligned}
& >(1+\varepsilon) W\left(D_{R}\left(N^{r-2}(v)\right)\right) \\
& >(1+\varepsilon)^{\frac{r}{2}} W\left(D_{R}\left(N^{0}(v)\right)\right) \\
& =(1+\varepsilon)^{\frac{r}{2}} .
\end{aligned}
$$

Case (2) $r$ is an odd number. In this case we have

$$
\begin{aligned}
8\left(r+\frac{1}{2}\right)^{2} & \geq W\left(D_{R}\left(N^{r}(v)\right)\right) \\
& >(1+\varepsilon) W\left(D_{R}\left(N^{r-2}(v)\right)\right) \\
& >(1+\varepsilon)^{\frac{r-1}{2}} W\left(D_{R}\left(N^{1}(v)\right)\right) \\
& =2(1+\varepsilon)^{\frac{r-1}{2}} .
\end{aligned}
$$

In both cases the above inequalities will not hold for sufficiently large $r$, the bound on $\bar{r}$, which is achieved when these inequalities are violated the first time, only depends on $\varepsilon$ and not on the order of graph $G$. By applying a similar argument used in [10], we can show $\bar{r} \leq c$, where $c=O\left(\frac{1}{\varepsilon^{2}} \ln \frac{1}{\varepsilon}\right)$. The proof is then proved.

The number of independent sets in $N^{\bar{r}}(v)$ is polynomially bounded in $\bar{r}$, and $\bar{r}$ is also polynomially bounded. By Lemma 3, a minimum Roman dominating set $D_{R}\left(N^{\bar{r}}(v)\right)$ can be computed in polynomial time $O\left(|V|^{c^{2}}\right)$, where $c=O\left(\frac{1}{\varepsilon^{2}} \ln \frac{1}{\varepsilon}\right)$. The following theorem shows the correctness and approximation guarantee of the algorithm.

Theorem 3. Algorithm $B$ returns a Roman dominating set $D_{R}=\bigcup_{i=1}^{k} D_{R}\left(N_{i}\right)$ whose weight is no more than $(1+\varepsilon) \gamma_{R}(G)$.

Proof. Let $D_{R}^{*}$ denote the optimal Roman dominating set. It is clear that $N\left(N^{\bar{r}_{i}}\left(v_{i}\right)\right)$ and $N\left(N^{\bar{r}_{j}}\left(v_{j}\right)\right)$ are mutually disjoint in $G$ for $i \neq j$, and $N\left(N^{\bar{r}_{i}}\left(v_{i}\right)\right) \cap$ $D_{R}^{*}$ dominates $N^{\bar{r}_{i}}\left(v_{i}\right)$. Thus by Lemma 5 we have

$$
\begin{aligned}
\gamma_{R}(G)=W\left(D_{R}^{*}\right) & \geq \sum_{i=1}^{k} W\left(D_{R}^{*} \cap N\left(N^{\bar{r}_{i}}\left(v_{i}\right)\right)\right) \\
& \geq \sum_{i=1}^{k} W\left(D_{R}\left(N^{\bar{r}_{i}}\left(v_{i}\right)\right)\right) \\
& \geq \sum_{i=1}^{k} \frac{1}{1+\varepsilon} W\left(D_{R}\left(N^{\bar{r}_{i}+2}\left(v_{i}\right)\right)\right) \\
& \geq \frac{1}{1+\varepsilon} W\left(\cup_{i=1}^{k} D_{R}\left(N^{\bar{r}_{i}+2}\left(v_{i}\right)\right)\right) \\
& =\frac{1}{1+\varepsilon} W\left(D_{R}\right) .
\end{aligned}
$$

Hence the theorem holds. 


\section{Conclusion}

In this paper we first show that the Roman domination problem in unit disk graphs is NP-hard, and then present a 5-approximation algorithm of linear time and a polynomial-time approximation scheme for this problem, respectively.

Grid graphs compose a subclass of unit disk graphs. However, hardness results for the Roman domination problem in grid graphs are still unknown (to the best knowledge of the authors). This raises an interesting problem for future work.

\section{References}

[1] E. J. Cockayne, P. A. Dreyer, S. M. Hedetniemi, and S. T. Hedetniemi: Roman domination in graphs, Discrete Mathematics. 278 (2004) 11-22

[2] P. A. Dreyer: Applications and Variations of domination in graphs, PhD Thesis, Rutgers University, New Jersey. (2000)

[3] A. Pagourtzis, P. Penna, K. Schlude, K. Steinhofel, D. Taylor, and P. Widmayer: Server placements, Roman domination and other dominating set variants, In Proceedings of the Second International Conference on Theoretical Computer Science. (2002) 280-291

[4] M. A. Henning: A characterization of Roman trees, Discuss. Math. Graph Theory. 22 (2) (2002) 325-334

[5] M. A. Henning and S. T. Hedetniemi: Defending the Roman Empire - A new strategy, Discrete Mathematics. 266 (2003) 239-251

[6] I. Stewart: Defend the Roman Empire!, Scientific American. December (1999) 136-138

[7] B. N. Clark, C. J. Colburn, and D. S. Johnson: Unit disk graphs, Discrete Mathematics. 86 (1990) 165-177

[8] H. B. Hunt III, M. V. Marathe, V. Radhakrishnan, S. S. Ravi, D. J. Rosenkrantz, and R. E. Stearns: NC-approximation schemes for NP-and PSPACE-hard problems for geometric graphs, Journal of Algorithms. 26 (2) (1998) 238-274

[9] M. V. Marathe, N. Breu, H. B. Hunt III, S. S. Ravi, and D. J. Rosenkrantz: Simple heuristics for unit disk graphs, Networks. 25 (1995) 59-68

[10] T. Nieberg, J. Hurink, and W. Kern: A robust PTAS for maximun independent sets in unit disk graphs, In Proceedings of the Thirtieth Workshop on Graph Theoretic Concepts in Computer Science. (2004) 214-221

[11] T. Nieberg and J. Hurink: A PTAS for the minimum dominating set problem in unit disk graphs, In Proceedings of the Third Workshop on Approximation and Online Algorithms. (2005) 6-7 